\title{
Contribution of Glucose Variability to HbA1C Levels in Patients With Type 1 Diabetes
}

Cardoso $L^{1}$, Batista $C^{1}$, Rodrigues $\mathbf{D}^{1,2}$, Barros $\mathrm{L}^{1}$, Moreno $\mathrm{C}^{1,2}$, Guelho $\mathrm{D}^{1}$, Vicente $\mathrm{N}^{1}$, Balsa $\mathbf{M}^{3}$, Martins $\mathrm{D}^{1}$, Oliveira $\mathrm{D}^{1}$, Carrilho $\mathrm{F}^{1}$

1. Department of Endocrinology, Diabetes and Metabolism, Centro Hospitalar e Universitário de Coimbra, Coimbra, Portugal
2. Faculty of Medicine, University of Coimbra, Coimbra, Portugal

3. Department of Endocrinology, Centro Hospitalar do Baixo Vouga, Aveiro, Portugal

\section{Background}

Optimal management of type 1 diabetes requires full understanding of the relationships between the triad: HbA1C, fasting plasma glucose, and glucose variability (GV). Total glucose exposure, including postprandial hyperglycaemia and glucose variability, should be considered in the evaluation of the patient's risk for complications. As GV may contribute to hemoglobin glycation we assessed the influence of GV in HbA1C levels.

\section{Research Design and Methods}

We retrospectively analysed 9,393 hours of continuous glucose monitorings (CGMs) from 61 patients with type 1 diabetes. Periods of 24 hours with missing values were excluded. We calculated various measures of GV and used a regression model to determine the impact of each GV measure to $\mathrm{HbA1C}$ level. GV was calculated using EasyGV ${ }^{\circ}$ software and CGMs were recorded using iPro' ${ }^{T M} 2$ (Medtronic, Northridge, CA).

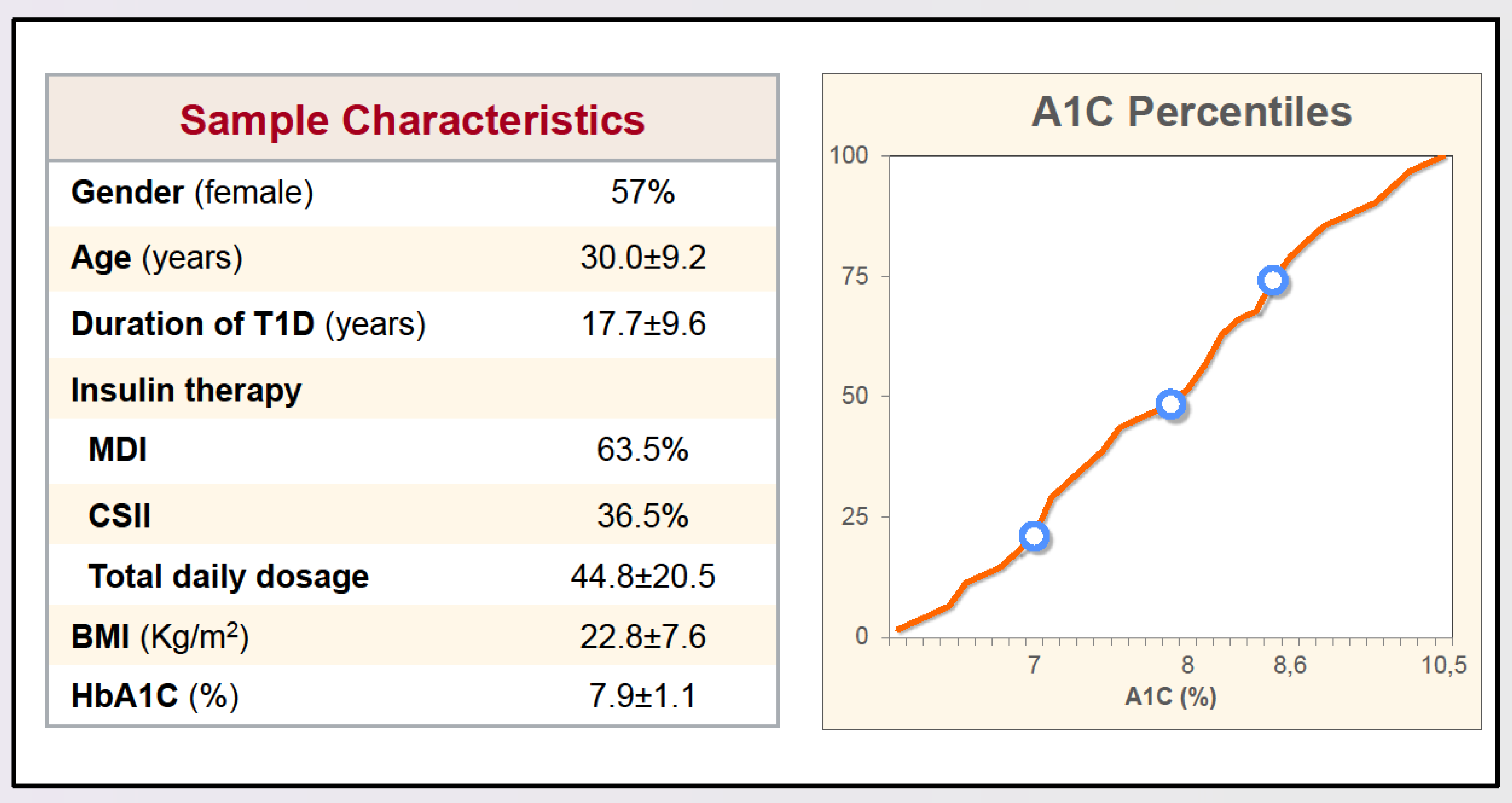

\begin{tabular}{|lccccc|}
\hline \multicolumn{5}{|c|}{ Glucose Variability } \\
\hline \multicolumn{5}{|c|}{ A1C (\%) } \\
SD & $<7 \%$ & $\mathbf{7 - 8} \%$ & $\mathbf{8 - 9} \%$ & $>\mathbf{9 \%}$ & $P$ \\
CONGA & $2.8 \pm 1.1$ & $3.4 \pm 0.8$ & $3.8 \pm 0.8$ & $4.24 \pm 1.1$ & $\mathbf{0 . 0 1 1}$ \\
MAG & $7.3 \pm 1.8$ & $7.4 \pm 1.6$ & $8.1 \pm 1.3$ & $8.8 \pm 2.0$ & 0.099 \\
LI & $2.2 \pm 0.9$ & $2.2 \pm 0.4$ & $2.5 \pm 0.5$ & $2.8 \pm 1.0$ & 0.104 \\
MAGE-CGM & $5.3 \pm 5.3$ & $5.4 \pm 2.3$ & $7.3 \pm 3.4$ & $9.3 \pm 6.1$ & $\mathbf{0 . 0 1 7}$ \\
MODD & $5.5 \pm 1.6$ & $6.6 \pm 1.5$ & $7.1 \pm 2.0$ & $8.2 \pm 4.1$ & 0.218 \\
ADDR & $3.1 \pm 1.1$ & $3.9 \pm 1.0$ & $4.1 \pm 0.9$ & $4.7 \pm 1.5$ & $\mathbf{0 . 0 4 1}$ \\
M-value & $21.1 \pm 11.5$ & $26.3 \pm 8.9$ & $32.6 \pm 9.9$ & $37.0 \pm 12.5$ & $\mathbf{0 . 0 1 2}$ \\
J-index & $12.9 \pm 7.3$ & $15.0 \pm 6.6$ & $16.5 \pm 7.3$ & $23.0 \pm 13.8$ & 0.333 \\
HBGI & $42.0 \pm 16.0$ & $46.0 \pm 18.0$ & $55.3 \pm 15.7$ & $66.8 \pm 26.6$ & $\mathbf{0 . 0 3 3}$ \\
GRADE & $8.7 \pm 5.3$ & $9.8 \pm 4.2$ & $12.0 \pm 4.1$ & $15.1 \pm 6.7$ & $\mathbf{0 . 0 4 1}$ \\
\%Hipoglycemia & $7.2 \pm 4.7$ & $7.0 \pm 3.7$ & $8.3 \pm 3.4$ & $9.9 \pm 5.6$ & 0.396 \\
\%Euglycemia & $10.9 \pm 13.0$ & $14.7 \pm 14.7$ & $9.3 \pm 9.9$ & $9.3 \pm 10.8$ & \\
\%Hiperglycemia & $5.1 \pm 3.0$ & $5.6 \pm 2.6$ & $5.5 \pm 2.3$ & $3.6 \pm 2.3$ & \\
\hline & $84.0 \pm 12.8$ & $79.7 \pm 15.5$ & $85.1 \pm 9.9$ & $87.1 \pm 10.9$ & \\
\hline
\end{tabular}

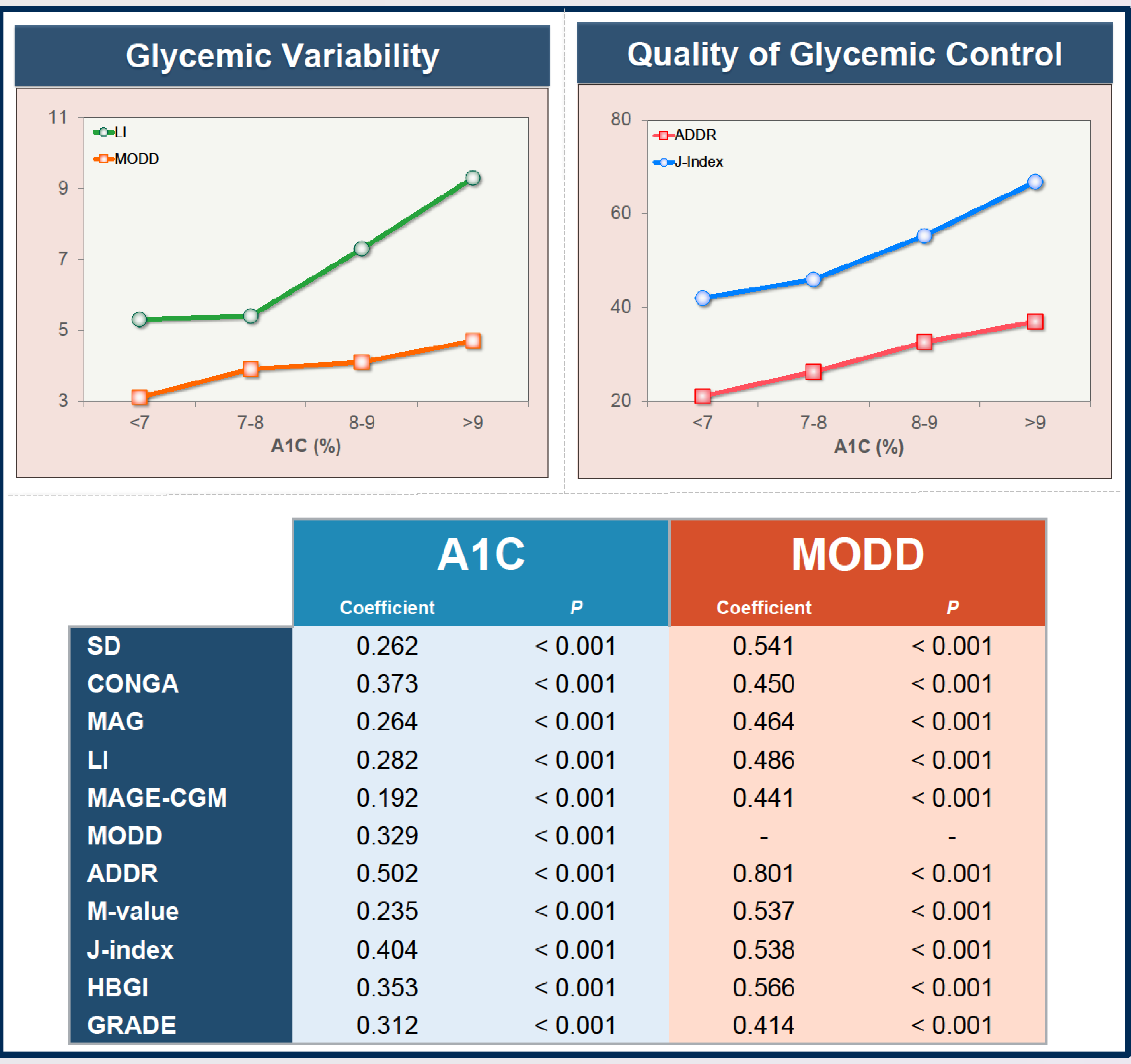

Contribution of Intra and Interday Variability to A1C Levels

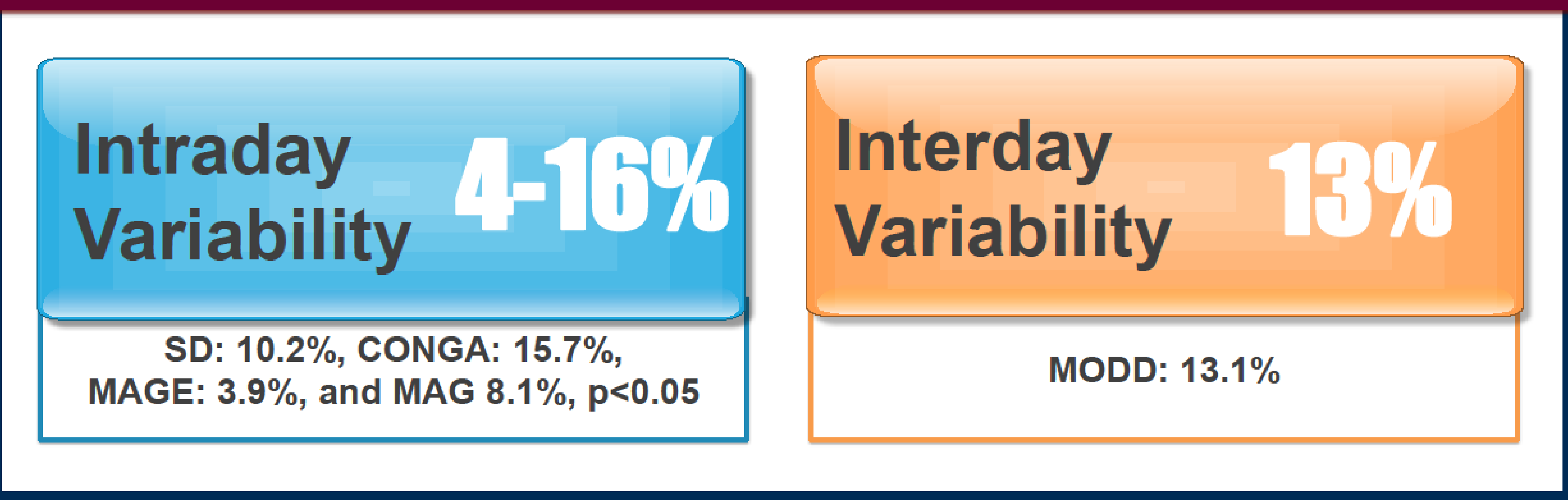

\section{Conclusion}

GV contributes significantly to $\mathrm{HbA} 1 \mathrm{c}$ levels. This effect is more pronounced at higher $\mathrm{HbA} 1 \mathrm{c}$ levels. Interday variability was the most important contributor to $\mathrm{HbA1C}$. GV impairs significantly the quality of glycemic control of type 1 diabetic patients. 